\title{
Le logement social à Lyon de 1906 à 1928. Principes et mise en œuvre d'un service public municipal des habitations à bon marché
}

\author{
Jérôme Henning
}

Droit et ville, $2020, \mathrm{n}^{\circ} 1$, p. 201-217

À Lyon, le problème du logement est aussi vieux que la ville elle-même ${ }^{1}$. Toutefois, la transformation de la ville suite à la Révolution industrielle a entraîné une urgence continue pour le développement d'un logement adapté aux plus pauvres.

$\mathrm{Au}$ début du XXe siècle, cette urgence n'est contestée par personne : groupements syndicaux, patronat, pouvoirs publics et institutions religieuses s'accordent sur la nécessité de développer des habitations salubres et à bon marché. Mais ces différents groupes se divisent sur les modalités de mise en œuvre de ces habitations. À Lyon, la particularité de cette lutte réside dans la position tenue par la municipalité. En effet, dès 1884, les conseils municipaux successifs tentent de développer un interventionnisme municipal - entendu comme l'ensemble des actions qui ont pour objet de peser sur l'évolution du système économique et social municipal - en matière de logement pour concurrencer l'initiative privée - entendue comme l'action d'une personne juridique en vue de la réalisation d'un besoin social que celle-ci considère comme nécessaire pour la collectivité ${ }^{2}$. Cet interventionnisme de la puissance publique caractérise la notion même de «logement social $»^{3}$ qui peut se définir comme « le logement qui a bénéficié pour sa réalisation de l'aide directe ou indirecte des pouvoirs publics, État et collectivités territoriales, et qui vise explicitement à loger les personnes à revenus modestes moyennant un loyer compatible avec leurs ressources $»^{4}$. Dans le cas de Lyon, le logement social se distingue ainsi du logement des plus pauvres en général et son origine est consécutive de l'intervention du législateur de 1894 et de 1906. D'un point de vue juridique, les critères de définition proposés sont restés stables malgré les interventions régulières du législateur et du juge sur la question. Le logement social se définit ainsi par la réunion de deux critères : le critère du financement et le critère de la pérennité de l'affectation sociale ${ }^{5}$. Dès lors, les définitions proposées convergent vers la détermination d'une mission de service public. Toutefois, au début du XXe siècle, l'échelon adéquat et le type de gestion pour la réalisation de cette mission de service public posent question. Ces interrogations, qui sont celles des édiles lyonnais, soulèvent

\footnotetext{
${ }^{1} \mathrm{~J}$. VERGELY, «Le logement populaire avant le XXe siècle », Le logement populaire à Lyon et sa région (18901940), t. 1, Société académique d'architecture de Lyon, 2015, p. 6-18.

${ }^{2}$ Pour une approche de l'histoire du logement social par le prisme de l'initiative privée : H. FROUARD, Du coron au HLM. Patronage et logement social (1894-1953), PUR, 2008, 187 p. et J.-P. FLAMAND, La question du logement, aujourd'hui en France, éd. Abeille et Castor, 2012, p. 36-60.

${ }^{3}$ Pour la banlieue parisienne, Emmanuel Bellanger a montré l'importance du rôle des municipalités dans le développement des Habitations à bon marché. L'auteur montre comment l'application des lois relatives au logement social profite aux communes grâce à deux vecteurs : d'une part la nécessité pour l'État de passer par les corps intermédiaires que constituent les communes et d'autre part la valorisation de la figure du maire bâtisseur (E. BELLANGER, «Les maires et leurs logements sociaux. Des années de fondation à l'âge d'or du grand ensemble (1920-1960)», Histoire urbaine, 2008, p. 95-107).

${ }^{4}$ J.-M. STÉBÉ, Le logement social en France, $3^{\mathrm{e}}$ éd., PUF, 2007, p. 7. Cette définition synthétise et modifie sur certains points la définition donnée par J.-P. FLAMAND (Loger le peuple. Essai sur l'histoire du logement social, La découverte, 1989, p. 11).

${ }^{5}$ C. DEBOUY (dir.), Droit et financement du logement social, éd. du Moniteur, 2012, p. 25-26.
} 
des enjeux liant la question du développement du logement social sur le territoire à celle de la décentralisation administrative ${ }^{6}$.

Dans cette perspective décentralisatrice, durant les mandats d'Édouard Herriot, maire radical de 1905 à 1957, la ville connaît son premier service public des habitations à bon marché (HBM). Avant 1905, la ville n'avait tenté que la construction privée d'HBM en accordant des exonérations fiscales ${ }^{7}$. L'élection d'Herriot coïncide avec l'adoption de la première loi accordant aux communes un moyen d'action en matière de logement social : la loi Strauss de 1906. De cette date jusqu'en 1928, c'est-à-dire jusqu'à l'adoption de la loi Loucheur, on constate que l'administration municipale tente de développer un service public des HBM pour limiter l'initiative privée au profit de l'interventionnisme public.

Pour bien comprendre cette dynamique, il faut revenir sur quelques aspects du problème du logement à Lyon. Au cours du XIXe siècle, la démographie lyonnaise connaît une hausse importante $^{8}$, notamment sous le Premier Empire. L'accroissement de la ville est consécutif du développement de l'industrie de la soie qui nécessite une main d'œuvre nombreuse. Cette hausse démographique se traduit par une hausse de la population ouvrière regroupée dans quelques quartiers à forte densité. Le nombre et la concentration de ces ouvriers - les canuts - inquiètent les pouvoirs publics qui craignent l'émergence d'un foyer révolutionnaire. Les quatre révoltes de 1831, 1834, 1848 et 1849 leurs donnent raison. Napoléon III a voulu régler ce problème ouvrier par des plans d'urbanisme nouveaux confiés au préfet Vaïsse. Ainsi en 1853, la plupart des quartiers ouvriers du centre-ville sont détruits par le percement de larges artères. Mais cette politique ne pense pas particulièrement le logement des plus pauvres dans la ville. Dès lors, lorsque la Commune éclate à Lyon, la question du logement salubre et à bon marché reste une revendication importante. Dans ce contexte, quelques initiatives privées de philanthropie et de patronage se développent. À cet égard, en construisant près de 2000 logements ouvriers entre 1886 et 1930, la société créée par Félix Mangini fait exemple ${ }^{9}$. En 1894, la loi Siegfried encourage les constructions privées mais les effets sur l'agglomération lyonnaise semblent assez faibles ${ }^{10}$. La difficulté réside dans le fait que les industriels lyonnais tentent de faire des bénéfices avec le logement ouvrier, conformément aux préconisations de Mangini, tout en refusant de construire des habitations qui seraient éloignées des ateliers. Or, ce dernier point apparait comme un frein au développement du logement social à Lyon par l'initiative privée car très peu de terrains sont disponibles à l'achat dans les quartiers historiquement ouvriers. À tel point qu'en 1905, les quelques sociétés privées créées disposent de fonds de réserve très importants sans possibilité de les investir ${ }^{11}$.

Face à cette inertie, le nouveau pouvoir municipal radical, incarné par Édouard Herriot, envisage de développer un service public des HBM. Après avoir gagné la bataille de la Séparation, le Parti radical tente de se renouveler autour de thématiques sociales ${ }^{12}$. À Lyon, cet

\footnotetext{
${ }^{6}$ P. QUILICHINI, Logement social et décentralisation, LGDJ, 2001, p. 9 et Y. JÉGOUZO, « Le logement social, entre solidarité, décentralisation, service public et concurrence », AJDA, 2008, p. 500.

${ }^{7}$ Le 18 août 1886, sur le fondement de la loi du 5 avril 1884, le Conseil municipal de Lyon a tenté d'encourager la construction d'habitations à bon marché par exonérations fiscales (A.M. Lyon, 481WP/1/1).

8 J. BIENFAIT, «La population de Lyon à travers un quart de siècle de recensements douteux », Revue de géographie de Lyon, Géocarrefour, 1968, p. 66-68.

${ }^{9}$ F. MANGINI, Les petits logements dans les grandes villes et plus particulièrement dans la ville de Lyon, Storck, 1891, 98 p. et P. CAYEZ, « Les petits logements dans les grandes villes. Lyon, 1886-1968 », Le mouvement social, 1986, p. 32.

${ }^{10}$ G. RICHAUD, « Du règlement sanitaire au plan d'extension : les origines du logement social public à Lyon », Le logement populaire à Lyon et sa région (1890-1940), t. 1, op. cit., p. 98.

${ }^{11}$ G. FRANCHE, Habitations à bon marché. Éléments de construction moderne, Dunod, 1905, p. 3. Le faible investissement de la Caisse d'épargne lyonnaise dans les sociétés d'initiative privée démontre le manque d'effets significatifs de la loi Siegfried dans l'agglomération (H. BIGET, Le logement de l'ouvrier : étude de la législation des habitations à bon marché en France et à l'étranger, Jouve, 1913, p. 292).

${ }^{12}$ S. BERSTEIN, Histoire du Parti radical, t. 1, PFNSP, 1980, p. 55-67.
} 
élan nouveau se traduit par une conception qui lie la question administrative et la question sociale $^{13}$. Le maire considère que seule une décentralisation réelle de la question sociale permet son règlement. Lyon s'engage ainsi pleinement dans la voie du socialisme municipal en procédant à des municipalisations de services publics concédés jusqu'alors. Cette branche radicale du socialisme municipal tente de concurrencer l'initiative privée dans tous les aspects de la vie administrative. Cela implique des créations de services publics nouveaux capables de répondre de façon décentralisée à la question sociale. Dans cette théorie sur le renouveau du pouvoir municipal, Herriot et son équipe s'emparent de la question du logement social. Entre 1906 et 1928, au gré des évolutions législatives, Lyon apparaît ainsi comme un véritable laboratoire du logement social afin de développer le municipalisme ${ }^{14}$.

Les travaux sur l'apparition du logement social à Lyon sont nombreux et reposent en majorité sur la sociologie ou sur les sciences de l'urbanisme ${ }^{15}$. Toutefois, pour ce qui concerne l'histoire du droit, l'étude de l'émergence du service public des HBM résulte de trois questionnements : $1^{\circ}$ la mesure sur le territoire des effets des lois adoptées en faveur du logement social ; $2^{\circ}$ la nature de l'incorporation du traitement du logement des plus pauvres dans le champ administratif dans une époque où le modèle étatique redéfini autour de l'État-

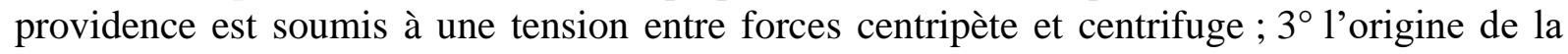
qualification juridique spécifique de «logement social» et la détermination d'un régime juridique propre.

Pour répondre à ces interrogations, il convient de rappeler que la notion de logement social, telle qu'elle est définie par les sociologues et par les juristes, renvoie à une intervention publique. À cet égard, le logement social se distingue donc du logement ouvrier, notion plus large qui peut relever d'une initiative privée répondant, par conséquent, aux lois économiques du marché. À Lyon, les exigences imposées par les considérations propres à une décentralisation en faveur des communes impliquent par conséquent la mise en œuvre d'un interventionnisme municipal en matière de logement des plus pauvres. Cet interventionnisme, parce qu'il vise à créer un service public, concurrence l'initiative privée et cherche à la réduire. La question demeure de savoir pour quelles raisons et comment, dans le contexte du socialisme municipal ponctué par l'adoption des lois Siegfried, Strauss, Bonnevay et Loucheur, la municipalité lyonnaise a tenté de freiner l'initiative privée afin de fonder un service public du logement social.

Les archives de la ville de Lyon, comme celles des différents acteurs du logement de cette époque et des offices créés au début du XXe siècle, montrent que le logement social lyonnais s'est construit en partie contre l'initiative privée par des campagnes vantant les mérites de l'interventionnisme public (I). Ces conceptions ont mené à une éviction progressive mais non totale des initiatives privées dans la mise en œuvre d'un service public municipal des HBM soumis, pour son développement, aux progrès législatifs (II).

\footnotetext{
${ }^{13}$ J. HENNING, Le radicalisme d'Édouard Herriot et la crise des institutions (1905-1954), Dalloz, 2019, p. 114165.

${ }^{14}$ Il faut noter la tenue du Congrès de l'exposition internationale d'hygiène urbaine tenue entre le 12 mai et le 17 juin 1907 au cours duquel la question du logement ouvrier a été centrale. Une partie du catalogue de l'exposition est consacrée aux expérimentations en matière de logement social (Exposition d'hygiène publique urbaine, Lyon, 1907, p. 22-32).

${ }^{15}$ C. BERTHET, Contribution à une histoire du logement social en France au XXe siècle. Des bâtisseurs aux habitants, les HBM des États-Unis de Lyon, L'Harmattan, 1997 et E. PASQUAL, Le logement populaire à Lyon dans l'entre-deux-guerres : l'Office public municipal d'Habitations à bon marché, Mémoire, Lyon II, 1987.
} 
$\left.\mathrm{I}^{\circ}\right)$ La promotion d'un interventionnisme municipal pour un logement social émancipé de l'initiative privée

Avant la Première Guerre mondiale, les différentes personnalités politiques lyonnaises semblent méfiantes à l'égard des sociétés créées depuis la loi Siegfried de 1894. Catalysant cette défiance, Herriot engage la municipalité dans une réflexion nouvelle sur le logement ouvrier et l'intervention publique (A) relayée par des réseaux de soutien en dehors de la mairie (B).

A - Les positions de la municipalité en faveur de l'intervention publique pour le développement du logement social

À partir de la décennie 1900, l'élection d'Herriot donne un élan aux idées favorables au logement social. Le nouveau maire tente de traduire ses conceptions dans une politique hygiéniste et décentralisatrice (1) en insistant sur la nécessité d'associer logement social et régie directe (2) excluant ainsi les acteurs privés potentiels.

faveur du logement social

1 - Entre hygiénisme et décentralisation : les conceptions d'Édouard Herriot en

Les conceptions d'Édouard Herriot sont articulées dès 1904 autour de l'hygiénisme et de la décentralisation, témoignant ainsi d'une conformité avec les préoccupations de son temps.

Tout d'abord, sur le plan de l'hygiène sociale, Herriot témoigne de l'influence des milieux hygiénistes sur la municipalité lyonnaise ${ }^{16}$. Au début du XXe siècle, il souhaite éradiquer deux fléaux : l'alcoolisme et la tuberculose. Selon lui, ces deux fléaux sanitaires sont consécutifs d'un mal logement, les logements étant étroits et insalubres. Le logement à bon marché doit être le moyen d'offrir de plus grands espaces d'habitat et de proposer des activités culturelles qui pourront réduire l'alcoolisme. De ce point de vue, le logement à bon marché ne concerne pas seulement les plus pauvres. Herriot insiste régulièrement, parfois contre une partie de sa majorité municipale, sur la nécessité d'offrir l'accès à ces logements aux familles nombreuses particulièrement concernées par ces méfaits ${ }^{17}$.

Ensuite, sur le plan de la décentralisation, Herriot considère que le règlement de la question sociale suppose une décentralisation au profit des communes ${ }^{18}$. Cette conception fonde la spécificité du socialisme municipal lyonnais. La commune étant une organisation humaine non artificielle, constituée du regroupement de familles autour d'une homogénéité économique, il appartient au pouvoir municipal de s'emparer des problèmes sociaux, sans attendre ni l'intervention de l'État, ni l'initiative des acteurs privés. Cette position vise à renforcer les prérogatives de la commune par rapport au département, considéré comme une circonscription bonapartiste aux mains du préfet, et surtout par rapport à l'État, considéré comme étant incapable d'appréhender les spécificités locales.

\footnotetext{
${ }^{16}$ S. FRIOUX, Les batailles de l'hygiène. Villes et environnement de Pasteur aux Trente Glorieuses, PUF, 2013, p. 239-254.

${ }^{17}$ Cette idée est relayée par Justin Godart, député et premier adjoint au maire avant 1914, qui prône un plus grand accès à la propriété afin de développer le type du « citoyen-propriétaire » (J. GODART, Travailleurs et métiers lyonnais, Cumin et Masson, 1909, p. 116-121). L'élargissement du logement social à des catégories de plus en plus vastes dépend d'un mouvement plus général de redéfinition de la question sociale à la fin des années 1890 (S. MAGRI, «Des "ouvriers" aux "citoyens modestes". Naissance d'une catégorie : les bénéficiaires des habitations à bon marché au tournant du XXe siècle », Genèse, 1991, n 5, p. 35-53).

${ }^{18}$ J. HENNING, op. cit., p. 122.
} 
Ce double postulat conduit Herriot à concevoir le service public des HBM comme devant relever exclusivement de la commune, sans possibilité de procéder à des concessions de service public.

\section{2 - La méthode de la régie directe envisagée par la mairie}

Pour mettre en œuvre les projets du maire, la municipalité lyonnaise tente un certain nombre d'expériences visant à fonder un service public des HBM affranchi des initiatives privées. Dans une ambiance résolument positiviste qui domine le Parti radical durant la Belle époque, la municipalité entend fonder son programme de logement à bon marché sur l'observation des modèles étrangers.

La première décision prise est de mener des voyages d'études auxquels participent le maire et les différents acteurs lyonnais. Le premier voyage les conduit en 1906 à Manchester puis à Glasgow ${ }^{19}$. De ce premier voyage, deux conclusions sont tirées : d'une part l'initiative privée n'est pas capable de prendre en charge la crise du logement d'une grande ville industrielle et d'autre part l'action communale doit reposer sur une société à caractère démocratique et philanthropique mais administrée en régie directe par la commune ${ }^{20}$. Pour les organisateurs du voyage, l'étude doit servir à contredire deux arguments opposés à l'interventionnisme municipal : les risques de paralysie des initiatives privées déjà entreprises et les risques de gaspillage des finances publiques par une mauvaise gestion ${ }^{21}$. Un deuxième voyage en Angleterre est organisé en 1912, afin de préparer la loi qui sera proposée par Bonnevay. Cette fois, le voyage d'études précise les souhaits de la mairie : le logement à bon marché devra prendre la forme, comme en Angleterre, du modèle de la cité-jardin ${ }^{22}$.

L'impact de ces voyages semble avoir été important pour le développement du logement social à Lyon. Ils servent en général de référence jusqu'en 1918 dans les délibérations du Conseil municipal. Toutefois, la méthode n'est pas fondée sur une véritable observation objective car les seuls voyages organisés ont pour destination le Royaume-Uni, ce qui indique qu'ils sont animés par un présupposé. Les modèles italiens, suisses ou néerlandais ne sont jamais évoqués alors même qu'ils sont connus et revendiqués par d'autres villes ${ }^{23}$. Il faut précisément retenir cette volonté d'inscrire le logement à bon marché lyonnais dans le sillage du modèle anglais qui favorise l'interventionnisme municipal.

À l'appui de ces conceptions novatrices, Herriot constitue en 1906 un réseau lyonnais préparant l'interventionnisme municipal qu'il souhaite mettre en œuvre.

\section{B - Les réseaux d'appui pour le développement du logement social lyonnais}

Le développement du logement social public à Lyon repose sur un réseau de personnalités militant pour un abandon du recours à l'initiative privée afin d'asseoir l'exclusivité de l'action publique. Dans ce vaste réseau, trois personnalités se détachent et vont

\footnotetext{
${ }^{19}$ On trouve un certain nombre de documents concernant ce voyage dans le fonds Herriot conservé à la Bibliothèque municipale de Lyon (BML, Fonds ancien, Ms 7529).

${ }^{20} \mathrm{Paul}$ Pic participe à ce voyage et s'en fait le traducteur engagé (P. PIC, « Un voyage social en Angleterre. Régies municipales - habitations ouvrières », Questions pratiques de législation ouvrière et d'économie sociale, 1907, p. 105-126).

${ }^{21}$ Ibid., p. 123.

${ }^{22}$ Ibid.

${ }^{23}$ P. PIC, «L'habitation ouvrière et la législation française », Alliance d'hygiène sociale. Congrès de Lyon, 13 16 mai 1907, p. 196-199.
} 
affirmer les principes concrets d'un service public des HBM : le médecin Jules Courmont (1), le juriste Paul Pic (2) et l'architecte Tony Garnier (3).

\section{1 - Jules Courmont, la faculté de médecine et les hygiénistes}

En initiant les thèses hygiénistes de la municipalité, les milieux médicaux lyonnais s'investissent en faveur du développement d'un logement salubre et à bon marché. Les médecins lyonnais, comme l'ensemble de leurs confrères français, participent à la promotion du logement social contre la propagation de la tuberculose. Toutefois, ils se distinguent par les rapports privilégiés qu'ils entretiennent avec la mairie.

Dans l'évolution du logement social lyonnais, Jules Courmont, professeur d'hygiène à la faculté de médecine entre 1900 et 1917, apparaît comme une personnalité importante. Ami d'Herriot, il collabore avec lui sur l'ensemble des questions de santé ${ }^{24}$. Entre 1900 et 1904, Courmont dirige une étude menée par Dupin et Lacomme qui démontre les rapports entre la tuberculose et le mauvais logement dans la ville de Lyon ${ }^{25}$. En faisant suite à cette étude, il dénonce en 1906, dans son Traité d'hygiène écrit avec Charles Lesieur, l'insuffisance de la législation sur la question du logement ouvrier ${ }^{26}$. On y décèle une critique de la loi Siegfried ne permettant pas un développement à grande échelle de logements ouvriers salubres et à bon marché. Entre le logement ouvrier issu de l'initiative privée et celui issu de l'intervention municipale, il semble que Courmont privilégie le second considéré comme plus économique et plus hygiénique, notamment en comparaison avec les logements construits par la Société de Mangini $^{27}$.

Son engagement apparaît encore lors des expositions internationales organisées à Lyon pour lesquelles il est commissaire général. L'exposition d'hygiène publique de 1907 et l'exposition internationale urbaine de 1914 sont orientées vers les découvertes de Courmont. Dans ces deux expositions, le logement social et ses évolutions sont présentés comme une obligation d'hygiène pour toute ville moderne.

2 - Paul Pic et ses disciples : le travail des juristes lyonnais en faveur du logement social

Un des autres appuis de la municipalité en faveur du développement d'un service public des HBM se trouve être la faculté de droit de Lyon. Sous l'impulsion d'un de ses professeurs, Paul Pic, la faculté encadre un certain nombre d'études qui offrent à la municipalité une documentation et des solutions juridiques pour favoriser le logement social. Ces études

\footnotetext{
${ }^{24}$ B. BENOIT, «Jules Courmont, militant hygiéniste », Lyon, centre du monde ! L'exposition internationale urbaine de 1914, Fage, 2013, p. 52-56.

${ }^{25}$ L'étude repose sur l'hypothèse d'un lien entre la catégorisation socio-professionnelle de certains quartiers et le nombre de décès dus à la tuberculose. Dupin et Lacomme établissent ainsi une comparaison entre le deuxième arrondissement, occupé en majorité par la bourgeoisie, et le troisième, plutôt peuplé par des familles ouvrières. En conclusion, l'étude démontre que dans le deuxième arrondissement les décès dus à la tuberculose sont de 24/10000 alors qu'ils sont de 41/10000 dans le troisième (P. DUPIN et L. LACOMME, Topographie de la tuberculose à Lyon : 1900-1904, Maloine, 1905, 406 p.). L'étude confirme à Lyon le lien qui avait été démontré à Paris par Paul Brouardel (Guerre à la tuberculose, Delagrave, 1903, 48 p. ; R. ROMME, « La crise actuelle des sanatoriums populaires », Revue scientifique, 1903, n 19, p. 264).

${ }^{26}$ J. COURMONT, Traité d'hygiène, t. 1, Baillières, 1906, p. 36.

${ }^{27}$ J. COURMONT, «Les œuvres lyonnaises d'hygiène sociale », Alliance d'hygiène sociale. Congrès de Lyon, 15-17 juin 1914, 1914, p. 366.
} 
s'inscrivent dans le soutien actif d'une partie des milieux juridiques lyonnais au socialisme municipal.

Paul Pic est l'un des pionniers de l'étude du droit du logement social en France. De manière générale, il se spécialise dans le droit du travail, soucieux d'offrir un cadre juridique aux mutations consécutives de la Révolution industrielle ${ }^{28}$. Paul Pic se réclame du Parti radical et du solidarisme. Bien avant le voyage de 1906 auquel il participe, le professeur lyonnais avait regretté l'absence d'initiatives publiques en faveur du logement ouvrier ${ }^{29}$. En 1894, il distingue trois types d'habitations à bon marché : celles consécutives d'une "spéculation pure », celles consécutives de «l'entreprise coopérative» et celles consécutives de «l'entreprise de patronage $»^{30}$. Des trois types de logements ouvriers, il explique que le type coopératif est le plus souhaitable, mais il démontre également que ce type ne pourra pas, à l'échelle d'une grande ville, loger tous les indigents. Par ailleurs, il estime que l'État doit interdire toute initiative de création de logement privé motivée par la spéculation. Pour cela, il considère qu'il faut interdire toutes les entreprises privées, même celles relevant du patronage et de la philanthropie, qui ne feraient pas apparaître dans leurs statuts une limitation stricte de la rémunération des capitaux engagés $^{31}$. À terme, Paul Pic souhaite une intervention prohibitive de l'État et une action constructive des communes.

Après 1909, ses conceptions se nuancent. Au sein des entreprises de patronage qui suscitaient sa méfiance, il distingue cette fois « le patronage proprement dit » du «patronage social » et «des sociétés philanthropiques $»^{32}$. Assez confiant dans les dispositions de la loi Strauss, il se dit favorable aux entreprises « de patronage social », c'est-à-dire constituées par les municipalités qui, selon lui, se substitueront peu à peu aux entreprises de «patronage proprement dit ». En 1909, son apport le plus décisif pour la formation d'un service municipal des HBM est son commentaire sur la loi Strauss en faveur de l'administration d'un tel service par la technique de la régie directe. Les tenants du socialisme municipal fondent leur administration sur cette technique administrative qui consiste à mettre fin aux concessions de service public. Selon eux, la régie directe constitue un mode d'administration plus démocratique et plus efficace tout en favorisant une décentralisation. À cet égard, Paul Pic plaide pour que Lyon ait la possibilité de créer et d'administrer en régie directe un service des $\mathrm{HBM}^{33}$.

L'autre apport de Paul Pic est moins théorique que pragmatique car il s'emploie à développer, à Lyon, un réseau de juristes susceptible de favoriser les théories d'un logement social municipal géré en régie directe. Durant les années 1900 et 1910, plusieurs thèses sont

\footnotetext{
${ }^{28}$ N. DOCKÈS-LALLEMENT, « Paul Pic », Dictionnaire historique des juristes français, PUF, 2007, p. 622-623, et « Paul Pic », La Faculté de droit de Lyon, 130 ans d'histoire, éd. lyonnaise d'art et d'histoire, 2006, p. 103107 ; N. HAKIM, « La science de la question sociale de Paul Pic ou les malheurs de l'hétérodoxie dans les facultés de droit », Le renouveau de la doctrine française, Dalloz, 2009, p. 123-158 ; D. BAYON et L. FROBERT, « Lois ouvrières et réformisme social dans l'œuvre de Paul Pic (1862-1944) », Le mouvement social, 2002, n 4, p. 5380 et « Paul Pic (1862-1944) et les lois ouvrières », Revue d'histoire des facultés de droit et de la science juridique, $1997, \mathrm{n}^{\circ} 18$, p. 69-94.

${ }^{29}$ P. PIC, Traité élémentaire de législation industrielle, Rousseau, 1894, p. 581-583.

${ }^{30}$ Ibid., p. 573.

31 À l'égard des entreprises de patronage, il précise que l'État doit veiller « à ce que les institutions patronales, destinées théoriquement à améliorer la condition de l'ouvrier, ne dévient pas de leur but, et ne servent point à masquer des spéculations coupables » (ibid., p. 587).

${ }^{32}$ P. PIC, Traité élémentaire de législation industrielle, $3^{\mathrm{e}}$ éd., Rousseau, 1909, p. 1051.

33 «Les communes et les départements, mis dans l'énumération donnée par la loi de 1894 des institutions appelées à coopérer à l'œuvre des HBM, sont formellement invitées, par la loi de 1906 (art. 6) à y participer financièrement. Le principe de la régie directe n’y est pas affirmé, comme dans les lois étrangères (...). Note : mais cette interdiction, conforme aux traditions du Conseil d'État, résolument hostile au développement des régies, paraît bien s'induire du texte. Cette interprétation restrictive est en tout cas celle à laquelle le ministre du travail s'est arrêté (...). Cette défiance à l'égard de l'État au regard des communes paraît tout à fait injustifiée ; tout au moins, conviendrait-il de leur reconnaître formellement le droit d'édifier en régie des habitations ouvrières sur les terrains à elles appartenant » (Ibid., p. 1060).
} 
soutenues en proposant des évolutions législatives favorables à l'intervention publique. Les collaborateurs de Paul Pic, comme Henri Duret ${ }^{34}$ et Émile Bouvier ${ }^{35}$, s'inscrivent au barreau de Lyon et enseignent à la faculté. On les retrouve durant toute la première moitié du XXe siècle dans les réseaux municipaux animant le logement social lyonnais.

\section{3 - Les conceptions architecturales de Tony Garnier}

Dans une autre perspective, il faut également évoquer l'importance de l'œuvre de Tony Garnier. Cet architecte, ancien de la villa Médicis et grand prix de Rome, s'installe à Lyon en 1905. On lui doit la plupart des grands bâtiments issus de la politique de transformation de la ville dans la première moitié du XXe siècle. Édouard Herriot et Tony Garnier partagent les mêmes vues : transformer Lyon pour construire une cité moderne, appelée cité industrielle par l'architecte, tournée vers le travail et le bien être ouvrier en répondant aux principes de la cité$\operatorname{jardin}^{36}$. Les différentes réalisations architecturales commandées par la mairie symbolisent d'ailleurs ce passage de la ville bonapartiste et bourgeoise à la ville républicaine et ouvrière.

L'utopie industrielle dessinée par Tony Garnier doit servir à loger l'ouvrier ${ }^{37}$. Pour autant, Garnier ne conçoit pas au profit de l'ouvrier un logement spécifique distinct du logement traditionnel. Ses conceptions reposent sur le principe du zonage, soit le découpage de la cité en zones fonctionnelles. Au centre de la cité, se situe la zone industrielle, c'est-à-dire les usines. Autour de ce centre, plusieurs zones correspondent aux différents besoins de la cité industrielle: placées hors de l'action des vents qui ramèneraient les fumées, les zones résidentielles sont formées selon des plans orthogonaux et sont traversées par une grande avenue destinée à recevoir des tramways. Ces zones résidentielles sont d'abord conçues pour faciliter la vie du travailleur. Au centre de cette zone résidentielle, apparaît une sous-zone administrative qui regroupe les bâtiments politiques et culturels servant l'ouvrier.

Ces différentes conceptions partagent toutes le même postulat: l'initiative privée ne peut pas répondre pleinement à la crise du logement ouvrier. Elles annoncent la mise en œuvre d'un service public du logement social.

\footnotetext{
${ }^{34}$ Sa thèse est interventionniste : «Après avoir passé en revue les résultats très intéressants auxquels est arrivée l'initiative privée, soit seule, soit avec le concours des pouvoirs publics depuis les lois de 1894 et de 1906, nous verrons qu'une intervention énergique des municipalités est nécessaire en matière d'habitation ouvrière à bon marché, parce qu'en fait, l'initiative privée seule est impuissante à assurer aux habitants, des logements en nombre suffisant et à des prix modérés, répondant aux exigences actuelles de l'hygiène (...). Quant à la législation française établie par la loi du 12 avril 1906, nous verrons qu'à défaut de l'intervention directe encore très discutée et d'ailleurs prohibée, elle permet aux municipalités d'intervenir par des modes très variés et, dans tous les cas, de prendre la direction du mouvement en vue de l'amélioration du logement ouvrier (...) (H. DURET, De l'intervention des municipalités en matière d'habitations ouvrières, Rousseau, 1910, p. 16).

${ }^{35}$ Notamment L'exploitation collective des services publics. Les régies municipales, Doin, 1910, $443 \mathrm{p}$.

${ }^{36}$ Les travaux de Tony Garnier s'inscrivent au sein des milieux favorables au logement social (G. BENOIT-LÉVY, « Lyon cité-jardin », Questions pratiques de législation ouvrière et d'économie sociale, 1907, p. 152-165).

37 T. GARNIER, Une cité industrielle, étude pour la construction des villes, Massin et Cie, 1917, 164 p. ; L. PIESSAT, Tony Garnier, PUL, 1988, 196 p.
} 
$\left.\mathrm{II}^{\circ}\right)$ La mise en œuvre du logement social à Lyon par la création d'un service public municipal des habitations à bon marché

La mise en œuvre d'un service public du logement social à Lyon peut paraître lente. En réalité, on constate que le développement du logement social lyonnais répond en grande partie aux évolutions législatives qui, malgré les revendications des édiles, n'offrent jamais assez de prérogatives à la municipalité (A). Toutefois, la Première Guerre mondiale va apparaître comme un accélérateur pour le logement social lyonnais (B).

A - La réception et l'évolution des lois sur le logement social au contact de la politique lyonnaise

La loi de 1894 était très critiquée parmi les radicaux et les tenants du socialisme municipal. La principale critique concernait la prohibition implicite qui était faite aux communes de construire et de gérer des logements à bon marché. Mais les évolutions législatives de 1906 (1) et de 1912 (2) ont ouvert la voie pour une réalisation des idées développées par Herriot et son entourage.

\section{1 - La difficile réception de la loi Strauss de 1906}

En 1902, avec l'arrivée d'une majorité radicale à la Chambre des députés, la réforme de la loi Siegfried est envisagée pour permettre aux communes de construire elles-mêmes des habitations à bon marché. Le Gouvernement d'Émile Combes crée ainsi en 1903 une commission interministérielle pour préparer cette réforme ${ }^{38}$. Mais il faut attendre 1906 pour que, suite à des pressions exercées par le Conseil municipal de Paris, les travaux de cette commission aboutissent à un projet de loi $^{39}$. Ce projet est déposé au Sénat où il est rapporté par Paul Strauss, sénateur radical. En fusionnant le projet gouvernemental et sa propre proposition de loi, Strauss prépare une réforme d'ampleur avec pour finalité la construction rapide et massive de logements sociaux. Malgré quelques résistances au Sénat, la loi est adoptée car les parlementaires semblent considérer que la méfiance à l'égard de l'interventionnisme municipal dans la loi de 1894 n'est pas justifiée ${ }^{40}$.

À Lyon, la réforme est reçue avec un grand intérêt. Mais malgré les espoirs de la municipalité, les projets entrepris rencontrent des difficultés juridiques qui reflètent la différence de vues entre l'État et la ville. La loi devait permettre aux communes de participer financièrement aux sociétés d'HBM sans pour autant leur permettre de se passer de cet intermédiaire. La municipalité lyonnaise interprète cette disposition de façon extensive en créant une société relevant seulement de capitaux municipaux et administrée en régie directe ${ }^{41}$. Dans un rapport établi le 17 juillet 1908, Herriot, avec l'appui de Pic, précise son projet : «J'avais pensé tout d'abord pouvoir vous proposer l'application des moyens prévus par la loi, mais l'étude à laquelle j'ai procédé, m'a montré les divers inconvénients qui résulteraient (...) de la mise en pratique des dispositions édictées par la loi. Une autre solution m'est apparue,

\footnotetext{
${ }^{38}$ JORF, Débats à la Chambre, 5 avril 1906, p. 1677.

${ }^{39}$ P. PIC, «L'habitation ouvrière et la législation française », op. cit., p. 207 et H. TUROT, « Les habitations à bon marché et la législation », Revue politique et parlementaire, 1907, n 51, p. 470-494.

${ }^{40}$ E. LANG, Les habitations ouvrières. L'action des communes et des départements, Imprimerie administrative, 1908, p. 20-39.

${ }^{41}$ A.M. Lyon, 1217 WP 171, p. 124. La ville cédait également des terrains à une société coopérative d'ouvriers (Bulletin officiel municipal de la ville de Lyon, 27 avril 1908).
} 
qui semble préférable à tous les points de vue. Cette solution consisterait à faire construire des habitations à bon marché par la ville qui les louerait à son profit ${ }^{42}$. Il s'agit de forcer la jurisprudence du Conseil d'État dans le silence de la loi de 1906 sur la question de la régie directe.

Ainsi, le 5 octobre 1908, le Conseil municipal adopte une délibération pour construire de nouveaux HBM en régie directe : «Vu le rapport par lequel le Maire expose (...) que (la loi) ne confère pas aux communes le droit de construire elles-mêmes des habitations et de les louer à leur profit, néanmoins aucune interdiction n'ayant été formulée par la législation française et le Gouvernement, rien ne s'oppose à ce que la ville de Lyon prenne l'initiative de tenter cette expérience sociale (...). Considérant que la construction et la location directe par la ville constituent une solution préférable à toutes les autres $(. ..){ }^{43}$. Mais le 19 novembre 1908 , contre ce montage, une circulaire du ministre du travail interdit le projet de la mairie jugé trop interventionniste. De plus, il est interdit à la municipalité de construire en régie directe des habitations sur les terrains qu'elle possède.

La création d'un service public des HBM se trouve ainsi compromise jusqu'en 1909 date à laquelle Herriot se résout à accepter l'interprétation de l'État et constitue la Société démocratique des habitations à bon marché de Lyon ${ }^{44}$. Le juriste lyonnais Émile Bouvier considérait que la loi de 1906 contraignait les communes à créer « une société paravent (...) qui, en réalité, était une filiale financière de la municipalité ${ }^{45}$. Cette Société regroupe, sous la présidence d'abord d'Édouard Herriot puis de Paul Pic des capitaux provenant de sociétés philanthropiques déjà existantes et de ceux de la municipalité. Pour éviter toute confusion avec la régie directe, la société sera gérée par un Conseil d'administration dirigé tout de même par les tenants du socialisme municipal et domiciliée à l'Hôtel de ville ${ }^{46}$. Mais les divergences de vues entre ces partisans de l'interventionnisme municipal et les acteurs philanthropiques plus anciens créent une inertie. Seuls quelques bâtiments sont transformés en HBM, mais presque aucune création ne voit le jour car la municipalité refuse de céder des terrains à cette société sur laquelle elle a pourtant la main. Elle redoute une réforme de la loi de 1894 qui exclurait de nouveau les communes de la création des HBM. Une telle réforme conduirait à céder la propriété des terrains municipaux donnés à la Société à des acteurs privés.

La réforme de 1906, même si elle résulte d'une volonté des partisans de l'interventionnisme municipal se trouve ainsi, par un défaut de conception, incapable de répondre aux attentes lyonnaises. C'est pourquoi, dès 1909, une grande campagne est organisée à Lyon pour obtenir une nouvelle loi.

\section{2 - L'heureuse évolution de la loi Bonnevay de 1912}

Entre 1909 et 1912, la municipalité lyonnaise mène une campagne pour convaincre les parlementaires de la nécessité de réformer la loi de 1894. Les parlementaires du Rhône travaillent ensemble à un certain nombre de projets, accueillent des congrès favorables au logement social public et organisent même plusieurs expositions internationales urbaines et hygiénistes dans lesquelles les insuffisances de la loi de 1894 sont pointées. Parmi les nombreux parlementaires du Rhône actifs sur la question, le maire de Lyon, Édouard Herriot, et le député du Rhône, Laurent Bonnevay, travaillent de concert.

\footnotetext{
${ }^{42}$ Ibid., 17 juillet 1908 ; H. DURET, op. cit., p. 340.

${ }^{43}$ A.M. Lyon, 1217 WP 170, p. 96.

${ }^{44}$ A.M. Lyon, 1217 WP 171, p. 604.

${ }^{45}$ É. BOUVIER, op. cit., p. 205.

${ }^{46}$ A.M. Lyon, 481 WP/1/1. Pour les affaires courantes, un administrateur délégué - Jo Costille - est nommé.
} 
La loi du 23 décembre 1912 est issue d'un compromis complexe entre un projet gouvernemental et plusieurs propositions de $\operatorname{loi}^{47}$. À l'issue des débats parlementaires, le législateur, contraint d'arbitrer entre les demandes d'un interventionnisme public plus affirmé et les craintes de trop grandes compétences laissées aux collectivités locales, propose la création d'établissements publics locaux ${ }^{48}$. Ces établissements publics locaux, appelés «office d'habitations à bon marché », répondent aux aspirations des communes tout en étant placés sous la tutelle de l'État.

Ce que l'on note, c'est l'investissement des parlementaires du Rhône et la victoire de leurs conceptions ${ }^{49}$. Cette fois, la loi de 1912 donne pleinement satisfaction à Herriot. Le 29 décembre 1913, le maire présente la politique de la ville sur la question en proposant que «Lyon soit une des premières villes à se doter d'un nombre significatif de logement destinés aux familles ouvrières et aux familles nombreuses $»^{50}$. Mais la guerre vient arrêter ce mouvement et il faudra attendre 1919 pour que le service public du logement soit systématisé.

B - La systématisation du logement social après la guerre

Dès 1919, Herriot considère qu'il faut enfin donner un élan concret et pérenne au logement social à Lyon, d'autant que la guerre a entraîné un accroissement de la population de la ville à l'inverse du reste du pays ${ }^{51}$. Afin de résoudre cette crise nouvelle du logement, des mesures provisoires sont prises : autorisation d'aménager les greniers en logement, création d'un office du logement centralisant les informations relatives aux logements vacants, et surveillance des spéculations illicites sur les loyers ${ }^{52}$. À plus long terme, il est envisagé la création d'un grand quartier (1) qui, en droit, nécessite que la mairie cède à la création d'un office public d'HBM (2).

\section{1 - Le projet du quartier des États-Unis ${ }^{53}$}

Afin de réaliser en pratique les élans donnés par l'organisation du logement à bon marché lyonnais, un vaste projet de construction est imaginé durant la guerre. Selon les vœux du Conseil municipal, ce grand quartier résidentiel, que l'on nomme quartier des États-Unis pour commémorer l'entrée en guerre des américains, est confié à Tony Garnier qui tentera d'y affirmer ses principes de « la cité industrielle ${ }^{54}$. L'objectif politique est double : d'une part, construire un nombre très important d'habitations pour mettre fin à la crise du logement et à l'augmentation des prix et, d'autre part, donner un signal fort de l'interventionnisme municipal en faveur des plus démunis. Le projet commence en 1917, Édouard Herriot et Tony Garnier

\footnotetext{
${ }^{47}$ JORF, Lois et Décrets, 25 décembre 1912, p. 10813 ; P. QUILICHINI, op. cit., p. 28-31.

${ }^{48}$ L. JÉGOUZO-VIÉNOT, Établissement public et logement social, LGDJ, 2002, p. 11.

49 Sauf une revendication en faveur des capacités d'emprunt des sociétés coopératives après le retrait d'un amendement de Godart à la Chambre des députés (JORF, Débats à la Chambre, 11 juillet 1912, p. 2274) et d'un d'Herriot au Sénat (JORF, Débats au Sénat, 12 décembre 1912, p. 1490).

${ }^{50}$ C. BERTHET, op. cit., p. 27. Afin de faciliter la construction rapide de logements, la municipalité accorde à la Société démocratique des Habitations à bon marché des dérogations au règlement relatif à l'hygiène (A.M., 1217 WP 174, p. 196).

${ }^{51}$ J. BIENFAIT, op. cit., p. 93.

52 E. PASQUAL, Le logement populaire à Lyon durant l'entre-deux-guerres : l'Office public municipal d'habitations à bon marché, op. cit., p. 34.

${ }^{53}$ Pour une étude complète sur le quartier des États-Unis, voir la thèse de Claire Berthet (op. cit.).

${ }^{54}$ Jusqu'en 1921, les différentes pièces administratives évoquent la « cité industrielle dans le quartier des ÉtatsUnis » montrant l'influence des conceptions de Garnier (A.M. Lyon, 481 WP/4). Ensuite, le Conseil municipal préfère l'expression de «Cité ouvrière » (délibération du 5 septembre 1921).
} 
envisagent un quartier construit de part et d'autre d'un axe central qui relie les différents immeubles à la gare de Vénissieux pour permettre une connexion rapide avec le centre de Lyon $^{55}$. Herriot impose que la construction se fasse sur une plaine inoccupée pour éviter les difficultés liées à d'éventuelles procédures d'expropriation. En 1920, les différents travaux préparatoires permettent de déboucher sur un premier projet de construction de 1730 logements permettant d'accueillir 12000 personnes. Le quartier comprend également 114 magasins, des écoles pouvant accueillir 2000 élèves et un certain nombre d'installations pour le bien être ouvrier : bibliothèque, piscine, stade, etc.

Cependant, deux problèmes apparaissent rapidement : celui du coût de la construction et celui de la gestion. La loi de 1912 autorise les communes à construire à leur frais, mais devant le montant des dépenses prévues -50 millions de francs -, Herriot espère une participation étatique. Mais cela suppose la création d'un office public municipal sur lequel la municipalité lyonnaise a toujours des réticences en raison de ses positions en faveur de la régie directe. L'autre difficulté réside dans la question de la gestion, car malgré les résistances de la mairie lyonnaise, la loi de 1912 n'autorise pas les communes à gérer directement les HBM. Pour ces raisons, la mairie lyonnaise se résout en 1920 à créer un office public des HBM conçu, non pas comme une œuvre de synthèse entre l'initiative privée et l'intervention publique, mais comme le bras armé de l'interventionnisme municipal.

\section{2 - La création de l'Office public municipal d'HBM}

Le 9 février 1920, dans un long rapport au Conseil municipal, le maire trace les grandes lignes de ce que doit être le logement social du XXe siècle. La création d'un office public d'HBM est réalisée par un décret du 29 mai 1920. L'office est alors chargé de la construction et de la gestion des immeubles. Puisqu'aux termes de la loi de 1912, les communes peuvent construire mais ne peuvent pas gérer, il est décidé qu'un seul et même organisme s'occupera des deux aspects. La commune abandonne ainsi sa volonté d'une construction en régie directe. Mais, afin que la commune ne perde pas trop la main, le Conseil d'administration est composé d'hommes favorables à la mairie. Parmi les 18 membres, on trouve entre autres Paul Pic, Louis Josserand et Isidore Cuminal. La mairie nomme 6 membres, la préfecture 6 autres et enfin les différents acteurs du logement social nomment les 6 derniers. Ce sont eux qui ont fait basculer l'office du côté d'Herriot au grand désarroi du préfet méfiant à l'égard du socialisme municipal. La présidence est donc confiée à Emmanuel Lévy, professeur à la faculté de droit et premier adjoint d'Herriot.

Pour les ressources, là encore les conceptions municipalistes entraînent une autonomie de l'Office par rapport à l'État. En réalité, il semble que la ville de Lyon est contrainte de doter l'office elle-même. En effet, les ressources fournies par l'État résultent de prêts pris sur les fonds de la Caisse des dépôts et consignations, mais que celle-ci peut refuser en cas de doute sur la capacité de remboursement des offices. Le fort interventionnisme municipal d'Herriot ainsi qu'un important prêt conclu durant la guerre conduisent la Caisse des dépôts à refuser les prêts demandés pour la construction des nouveaux logements ${ }^{56}$. Face au refus de la Caisse des dépôts, la commune propose de former un fond de garantie destiné à obtenir des prêts et, pour

\footnotetext{
${ }^{55}$ Délibération du 29 mars 1917 (A.M., 1217 WP 177, p. 106).

${ }^{56}$ On remarque que ce refus de la Caisse des dépôts s'inscrit dans une réticence générale de l'organisme à l'égard des projets de logements ouvriers (A. FOURCAUT, « La Caisse des dépôts et consignations et le logement social. De la loi Loucheur à la guerre : un constat d'échec ?», La Caisse des dépôts et consignations, la Seconde guerre mondiale et le XXe siècle, Albin Michel, 2003, p. 185-196). Il faut toutefois apporter quelques nuances en fonction des époques (H. FROUARD, «La Caisse des dépôts et les HBM (1894-1921) », Histoire urbaine, 2008, $\mathrm{n}^{\circ} 23$, p. 23-40).
} 
le court terme, à doter l'office public municipal de 110000 francs employables immédiatement ${ }^{57}$. Les dépenses ordinaires, frais d'entretien et de personnel, sont supportés par la commune qui donne une subvention annuelle correspondante. On constate ainsi qu'au début de l'office d'HBM lyonnais les ressources sont exclusivement municipales. Ni l'État, ni l'initiative privée ne participent à cette campagne du logement social, alors même qu'après 1918, il existe encore neuf sociétés coopératives et dix sociétés anonymes.

Avec le vote de la loi Loucheur en 1928, l'engagement de l'État augmente et l'office public municipal perd progressivement ses liens directs avec l'administration communale ${ }^{58}$. À Lyon, la réception de cette loi entraîne un bouleversement dans le service public du logement social. Après 1928, la ville poursuit ses projets, notamment dans le quartier des États-Unis, mais il faut constater que le département devient le premier acteur du logement social.

À travers l'étude du service public des HBM, on peut mettre en évidence trois phénomènes. Premièrement, l'étude des effets des lois adoptées entre 1906 et 1928 peut montrer comment l'évolution législative en faveur du logement social se construit dans un dialogue avec les collectivités. La réforme de la loi Strauss par la loi Bonnevay résulte ainsi en partie de la constatation sur le territoire de l'insuffisance des mécanismes juridiques prévus. Deuxièmement, il faut insister sur le lien démontré par les juristes entre décentralisation et logement social. À Lyon, les revendications en faveur d'un service public municipal des HBM relève en partie de tentatives d'extension des prérogatives des collectivités locales dans une tension entre le centre et la périphérie. Enfin, troisièmement, quant à la question de l'origine de la qualification du logement social, il convient d'admettre que c'est à partir de la loi Strauss que l'on distingue une catégorie juridique de l'intervention publique en matière d'HBM distincte de l'initiative privée. Toutefois, il faut attendre la loi Bonnevay pour que, à Lyon, on puisse observer la constitution d'un service public à part entière disposant de règles de financement, de fonctionnement et de gestion propres à la réalisation de l'objectif social qui lui est assigné.

\footnotetext{
${ }^{57}$ En réalité, la somme est assez faible. En 1921, une correspondance entre Édouard Herriot et Tony Garnier révèle les difficultés de la municipalité qui est incapable de gager les sommes avancées pour les travaux des maisonstypes (1 326000 francs) entamés par Garnier (A.M. Lyon, 481 WP/4).

${ }^{58}$ B. POURTOUT, «La première loi de programmation de logements sociaux : la loi Loucheur et les offices publics d'HBM », Villes de banlieue. Personnel communal, élus locaux et politique urbaine en banlieue parisienne au XXe siècle, Créaphis, 2008, p. 109-118.
} 